\title{
SUTURELESS THYROIDECTOMY VASUCULAR CONTROL USING BIPOLAR ELECTROTHERMAL CAUTERY.
}

Challa. S, Sushama Surapaneni.

1. Professor, Department of Surgery, Konaseema Institute of Medical College. Amalapuram. Andhra Pradesh,

2. Associate Professor, Department of Surgery, Konaseema Institute of Medical College. Amalapuram. Andhra Pradesh.

\section{CORRESPONDING AUTHOR}

Sushama Surapaneni

Department of Surgery,

KIMS, Amalapuram, Andhra Pradesh,

E-mail: sushama720@gmail.com

Ph: 00919849180856.

ABSTRACT: INTRODUCTION: One of the routinely performed surgery is thyroidectomy. Rates of complications have decreased because of new technologies that are being invented likeligasure, harmonic scalpel and bipolar surgical diathermy. In this study we used bipolar surgical diathermy for thyroidectomy instead of ligasure and harmonic scalpel which are costly and available in higher centers only. MATERIALS: 40 patients with thyroid swelling attending surgical OPD between the period of 2010-2012 were selected. Proper preoperative workup was done local ethical committee approval was taken .thyroidectomy done using bipolar surgical diathermy .patients were reviewed for any complications following thyroidectomy likehaemorrhage, hypocalcemia and recurrent laryngeal nerve palsy. RESULTS: Out of 40 patients who underwent sutureless thyroidectomy none of them had any primary, secondary or reactionary haemorrhage. One patient who underwent total thyroidectomy for follicular carcinoma had transient hypocalcaemia .

KEYWORDS: Thyroidectomy, bipolar surgical diathermy, hypocalcemia, recurrent laryngeal nerve palsy, haematoma.

INTRODUCTION: Thyroidectomy is one of the most common operations performed for thyroid diseases throughout the world and has an extremely low morbidity rate. First thyroid surgery accounts back to $12^{\text {th }}$ century, but with extremely high mortality and morbidity for which it was not routinely performed (1). With the advent of general anesthesia and antisepsis mortality rates were reduced to $8 \%$ by $19^{\text {th }}$ century. As the mortality rates decreased and surgical techniques have advanced the complications with thyroid surgery such as recurrent laryngeal nerve injury and hypocalcaemia became more apparent which are reported as 5-15\% (2)(3). Rates of complications and morbidity in thyroid surgery have decreased greatly because of new technologies that are being invented like ligasure, harmonic scalpel and bipolar surgical diathermy.

Most of the studies have been performed comparing ligasure and suture knot tying or harmonic scalpel with conventional knot tying. In this study we choose to use bipolar electrocautery for performing thyroid surgeries that is sutureless thyroidectomy (Lobectomy, Neartotal thyroidectomy and Subtotoal thyroidectomy)

MATERIALS AND METHOD: MATERIALS: 40 patients with thyroid swelling attended surgical OPD between the period of 2010-2012. Out of which 22were solitary thyroid nodule,4 multinodular goiter,7carcinoma and 7 thyrotoxicosis. A complete pre-operative work up which 
included biochemical, ultrasonography, IDL , Fine needle aspiration cytology was performed and CT scan was done for FNAC proven cases of malignancy. At Local ethical committee approval was taken.

METHOD: After endotracheal intubation under general anaesthesia patient was positioned in reverse trendelenberg position. Kochers neck incision was made extending from one sternocleidomastoid to other. Upper and lower flaps raised upto thyroid notch and sternalnotch. Deep fascia was incised vertically in midline. Strap muscles cut at the junction of upper $1 / 3^{\text {rdandlower }} 2 / 3^{\text {rd }}$ border and retracted depending on the size of the gland. 2 tributaries of middle thyroid vein were identified and cauterized with bipolar electrothermal cautery. Superior thyroid pedicle was dissected all terminal branches of superior thyroid artery , inferior thyroid artery and other unnamed vessels were cauterized with bipolar cautery. Recurrent laryngeal nerve was identified on both the sides and preserved and parathyroid glands were identified at least on one side and preserved in all possible conditions. Proper haemostasis was achieved. Romovac suction drain kept in all cases. Strap muscles and deep fascia were sutured with vicryl, platysma was sutured with chromic catgut. Skin margins approximated with monocryl or 5-0 prolene. Suction drain was removed after 48 hours, patient was given a broad spectrum antibiotic for 5 days and discharged on $5^{\text {th }}$ postoperative day.

Follow up of the patients was after 1 week, 6weeks and 12weeks for any recurrent laryngeal nerve palsy, hypocalcaemia.

RESULT: Out of 40 patients who underwent sutureless thyroidectomy none of them had any primary, secondary or reactionary haemorrhage. One patient who underwent total thyroidectomy for follicular carcinoma had transient hypocalcaemia and was treated with Vit D, calcium gluconate and on regular follow up it was normal. Now the patient is not on any medication.

DISCUSSION: Thyroid surgery can be performed safely with the appropriate technique, meticulous haemostasis and precise knowledge of the anatomy (4).Bleeding is a major complication of thyroid surgery both intraoperative and postoperative with the potential to cause life threatening airway obstruction, haemodynamic shock andeven death. Sometimes it requires reoperation and may prolong the operation and stay. During thyroidectomy bleeding can obscure the operative field making safe dissection of recurrent laryngeal nerve and parathyroid gland difficult and prolonging the operation time. Effective vessel haemostasis can be achieved by suture knot tying technique or newer techniques of vessel haemostasis like harmonic scalpel, ligasure (alternative bipolar surgical diathermy system),clips, staples, tissue adhesives haemostatic agents/sealants, monopolar and bipolar surgical diathermy. The safest, most efficient and cost effective way to achieve haemostasis is hotly debated. During the last decade alternative techniques for improving safety and effectiveness of thyroidectomy have been developed. The conventional suture knot tying technique requires a large number of surgical ties and is time consuming ,decreases healing as well as increases wound infection, requires good exposure, injury to neighboring structures and foreign body reaction(5). Harmonic scalpel uses ultrasound technology to denature protein in the vessel wall and tissues upto $5 \mathrm{~mm}$ thick leading to coagulation (6). A major criticism to harmonic scalpel comes from its cost, it is disposable and expensive (7). Ligasure vessel sealing device utilizes both electrical and elastin in vessel walls and tissue upto $7 \mathrm{~mm}$ in diameter to provide haemostasis(8).use of the 
ligasure in thyroid surgery did not significantly reduce operative time, blood loss or complication rates compared with conventional knot tying but it increased operative $\operatorname{cost}(9)$. The use of metal clips has a wide acceptance among surgeons to achieve haemostasis. However they can cause significant interference with computed tomography and may have poor holding power(10). They can erode into important anatomic structures and may cause tearing of the adjacent vessels. A monopolar diathermy can be applied quicklyandsafely to vessels $<2 \mathrm{~mm}$ in diameter but the extension of the lateral thermal energy it produces cannot be estimated precisely so it can cause inadvertent thermal injury to adjacent structures(11). An alternative to conventional surgical diathermy is bipolar surgical diathermy. The path of the current can be constrained to pass only through the tissues being treated. This is achieved using a special forceps in which the two halves of the instrument are insulated from one another and in effect one half becomes the source of current andthe other destination. A small blood vessel gripped between the jaws will be coagulated whereas there will be no damage to the adjacent structures, most of the modern diathermy machines support the use of bipolar probe without modification unlike ligasure and harmonic scalpel in which the machine is very costly.

CONCLUSION: The use of bipolar surgical diathermy is a better choice for thyroidectomies as compared to other techniques due to its cost effectiveness, less intraoperative and post operative complications rate.

\section{REFERENCES:}

1. Giddings A. The history of thyroidectomy. J R Soc Med.1998;91(Suppl 33):3-6.

2. Snyder S, Hamid K, Roberson C, et al. Outpatient thyroidectomy is safe and reasonable: experience with more than 1,000 planned outpatient procedures. J Am Coll Surg. 2010; 210(5):575-82, 582-4.

3. Abboud B, Sleilaty G, Mallak N, et al. Morbidity and mortality ofthyroidectomy for substernal goiter. Head Neck. 2010;32(6):744-9.

4. Bliss R. D., Gauger P. G., Delbridge L. W. Surgeon's approach to the thyroid gland : surgical anatomy and the importance of technique.World J Surg, 2000, 24 (8) : 891897.

5. Vesely D. L., Angtuaco E. J., Boyd C. M. Sinus tract in the neck :a rare complication of subtotal thyroidectomy for Graves disease J Med, 1986, 17 (3-4) : 253-261.

6. Ethicon.Hamrmonic.Focus.http://www.harmonic.com/dtcf/pages/HarmonicFocu s.htm. Accessed 10 March 2010.

7. Emanuele Ferri,Enrico Armato,Giacomo Spinato,and Roberto Spinato :Focus Harmonic Scalpel Compared to Conventional Haemostasis in Open Total Thyroidectomy: A Prospective Randomized Trial International Journal of OtolaryngologyVol 2011 (2011),Article ID 357195, 7 pages doi:10.1155/2011/357195

8. Valley Lab. Technology. http://www.ligasure.com/ligasure/pages.aspx. Accessed 10 March 2010.

9. Kiriakopoulos A., Dimitrios T., Dimitrios L. Use of a diathermy system in thyroid surgery. Arch Surg, 2004, 139 (9) : 997-1000. 
10. Klein R. D., Jessup G., Connolly R. J., Schwaitzberg S. D. Comparison of titanium and absorbable polymeric surgical clips for use in laparoscopic cholecystectomy. Surg Endosc, 1994, 8 (7) : 753-758.

11. Sengupta S., Webb D. Use of a computer-controlled bipolar surgical diathermy system in radical prostatectomies and other open urological surger

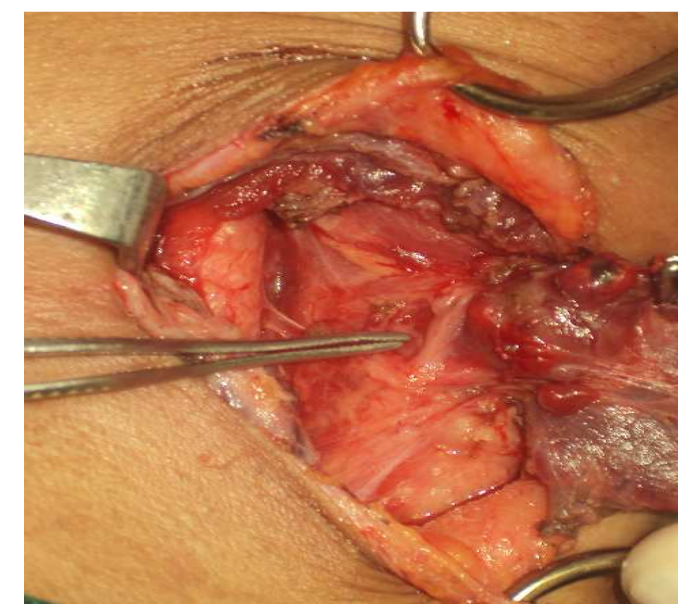

\section{Photograph showing recurrent laryngeal nerve}

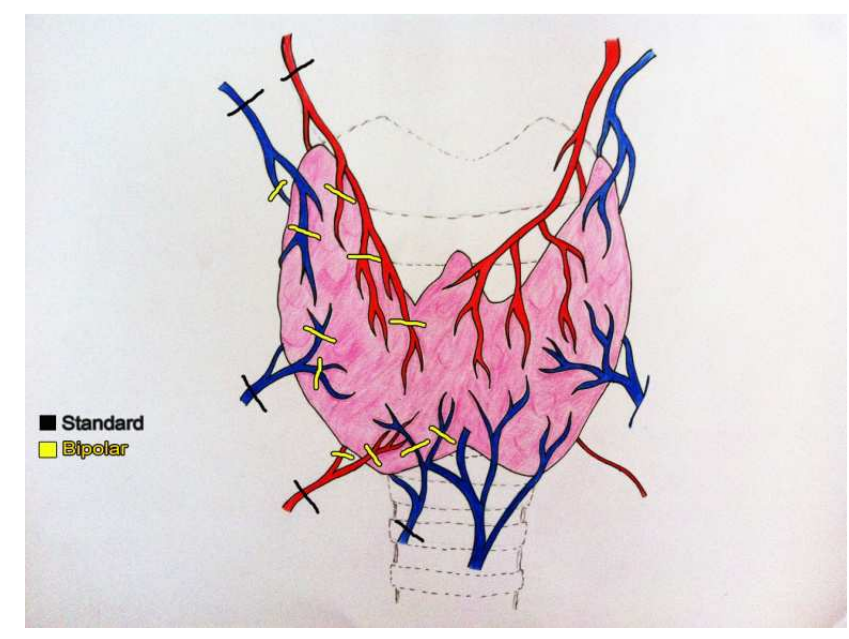

Schematic diagram showing difference between standard knot tying and bipolar surgical diathermy 